\title{
Two-Dimensional Kinetic Shape Dynamics: Verification and Application
}

\author{
Ismet Handzic, ${ }^{1}$ Haris Muratagic, ${ }^{2}$ and Kyle B. Reed ${ }^{2}$ \\ ${ }^{1}$ Tao Life Sciences, Greenville, SC, USA \\ ${ }^{2}$ The University of South Florida, Tampa, USA \\ Correspondence should be addressed to Kyle B. Reed; kylereed@usf.edu
}

Received 4 May 2016; Accepted 25 September 2016

Academic Editor: Thordur Runolfsson

Copyright (c) 2016 Ismet Handzic et al. This is an open access article distributed under the Creative Commons Attribution License, which permits unrestricted use, distribution, and reproduction in any medium, provided the original work is properly cited.

\begin{abstract}
A kinetic shape (KS) is a smooth two- or three-dimensional shape that is defined by its predicted ground reaction forces as it is pressed onto a flat surface. A KS can be applied in any mechanical situation where position-dependent force redirection is required. Although previous work on KSs can predict static force reaction behavior, it does not describe the kinematic behavior of these shapes. In this article, we derive the equations of motion for a rolling two-dimensional KS (or any other smooth curve) and validate the model with physical experiments. The results of the physical experiments showed good agreement with the predicted dynamic KS model. In addition, we have modified these equations of motion to develop and verify the theory of a novel transportation device, the kinetic board, that is powered by an individual shifting their weight on top of a set of KSs.
\end{abstract}

\section{Introduction}

Throughout ancient and modern history, the mechanism of rolling has been both useful and fascinating. The dynamics of a circular object rolling down a slope is an elementary and age-old mechanics concept and is often taught in early mechanics courses. The equations of motion of a two dimensional circular object rolling down a slope is a common physics problem. However, a notion that has gained less attention is the forced rolling of irregular objects or curves. The equations of motion that exactly dictate the rolling of a shape as a known force input is applied to its rotational axle have not previously been developed.

Dynamically, the behavior of such an asymmetric shape under forced conditions is similar to a circular shape rolling down a ramp, as is shown in Figure 1. In all three instances, the rolling object is not in static equilibrium due to an unbalanced moment created by the applied force at the axle point. The rolling kinematics $\left(d^{2} \theta / d t^{2}\right)$ of a circular wheel is created by a decreasing rolling surface height with distance traveled $(d y / d x)$, while the same rolling motion is created for a rounded object by a decreasing shape radius around the object (relative to the axle point) $(d R / d \theta)$. This generalized comparison is seen in

$$
\frac{d^{2} \theta}{d t^{2}} \propto \frac{d y}{d x} \propto \frac{d R}{d \theta} .
$$

Although we do have firm knowledge of how to exactly predict the behavior of a wheel rolling down a ramp as a force is applied to its axle (Lagrangian, Energy Method, etc.), the derivation of an arbitrary and asymmetric rolling curve in a similar situation has largely been ignored.

The concept and approach of the kinetic shape (KS) [1] offer a way to analyze these types of dynamics problems. The KS equation defines a smooth shape in static equilibrium that can produce exact and predictable reaction forces when forced onto a flat surface. The related concepts and background of the KSs are discussed in Section 2.

Section 3 shows the derivations for the kinematic equations that predict the angular kinematics of a two dimensional KS rolling freely with a force applied to its axle. This section also extends the KS concept from predicting a KS's static behavior to deriving its dynamic behavior. Although these derived equations of motion are used for KSs, they can be 


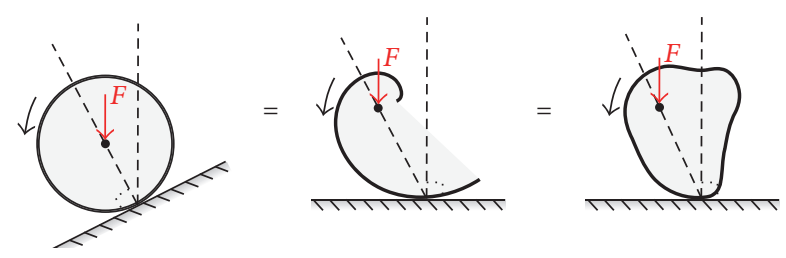

FIgURE 1: The rolling dynamics of a circular wheel on a decline can be equivalent to that of a shape with a negatively changing radius.

applied to predict the rolling dynamics of any smooth object with a force applied at its axle and onto a flat surface. Along with our derivation of formulas to predict the angular and linear motion of a rolling KS, we also present a physical experiment to verify these equations in Section 4.

These derived rolling dynamics equations are then modified in Section 5 to create and analyze the motion of a novel locomotion and recreation application, the kinetic board. The kinetic board allows a user to be able to initiate and continue linear overground motion by simply shifting their weight onto a set of predefined KSs, utilizing their weight as a source of power.

\section{Background}

The Archimedean spiral (similar to the involute of a circle) was first studied by Archimedes in 200 B.C. after being inspired by fellow mathematician Conon of Samos and his work on conic sections [2]. Conic sections are curves created by the intersection of cones, often producing interesting, smooth, and asymmetric curves (e.g., Archimedean spiral, logarithmic spiral, Cortes' spiral, lituus, etc.). Although these curves have several variations, the spirals, such as the Archimedes spiral, are particularly unique because they represent a curve of radius that increases with angle in polar coordinates. In a physical sense, this allows a spiral to roll on a flat surface much like a circular wheel rolls down an incline (Figure 1). A physical spiral is beneficial and is utilized in several applications including cam design [3], microbiology [4], and fluid dynamics [5] and can be a great tool in mechanical design situations where passive or forced rolling is desired.

One study that harnesses this unique passive rolling principle is a two-dimensional crawling and deformable robot that is able to roll by manipulating its outside rim to attain a nonconstant radius [6]. The robot's shape is mechanically deformed by shortening and lengthening internal chords that are attached to a rolling surface, causing it to roll towards the decreasing radius on a flat or slopped surface (Figure 2(a)). Its shape can be adjusted to move the robot both forward and backward depending on which chords are pulled. Although this study demonstrates passive rolling of a rounded shape, it lacks an analytical solution for this motion and how it is affected by the robot weight or ground reaction forces while rolling.

Another robot design that utilizes spirals as a means of locomotion is illustrated in Figure 2(b). This quadruped walking robot has compliant Archimedean spiral feet used to vary its foot compliance by altering the orientation of the spiral feet in order to minimize the robot's energy expenditure [7]. However, neither the kinetic nor kinematic effects of this foot design are addressed.

Other spiral curves roll when a force is exerted onto their eccentric rotational axle. Cams take a rotary kinematic input and transfer it to a cam follower as a linear kinematic output. The change in radius of a cam determines the exact linear output motions of the cam follower. Prior research on cams and cam followers have typically focused on the transfer of motion and the friction involved and do little to address cam shapes as a force input source to produce predicted rolling forces or motions $[3,8]$. Cams are not generally designed to produce predefined input and output kinetics of the cam or the cam follower.

In machine systems and mechanical design, studies of gears and pulleys consider different types of dynamics within mechanisms, including forces between gear or pulley teeth and their elastic behavior as they are engaged $[9,10]$. Although these studies include the kinematics of circular and elliptical gears and drive shapes, they do little to address the effects of forced rolling of asymmetric shapes [11, 12].

Another mechanics study related the exact meshing relationship of square and irregularly shaped wheels on matching unevenly shaped roads (similar to a rack and pinion) [13]. Although this study discusses the rolling kinematics of the irregular shapes to its predefined uneven road, the dynamics forces acting on such a system were not taken into account.

In mathematics, there have been articles centered on roulettes, which are curves traced by points on a twodimensional circular or noncircular shape while rolling over flat or uneven surfaces. These provide insight to the kinematics of these shapes and the roulette curves that they produce but do not focus on any physical kinetics of these shapes when rolling over various surfaces [14].

2.1. Kinetic Shapes. Kinetic shapes (KS) are smooth shapes that can produce a predicted reaction force parallel to the ground. Note that a KS may, but is not necessarily, defined as a spiral such as an Archimedean spiral. The two-dimensional KS equation (2) allows for a two dimensional shape $(R(\theta))$ to be derived in polar coordinates, given the applied forces perpendicular $\left(F_{v}(\theta)\right)$ and parallel $\left(F_{r}(\theta)\right)$ to a flat surface $[1,15]$. The parameters of $(2)$ can be visualized in Figure 3

$$
R(\theta)=R\left(\theta_{i}\right) \exp \int \frac{F_{v}(\theta)}{F_{r}(\theta)} d \theta .
$$

A KS is useful in that it can be utilized to create a desired shape that will yield a predefined kinetic behavior of the shape. The KS concept can be used anywhere that exact position-dependent force redirection is needed, which includes the fields of musical acoustics [16], rehabilitation engineering [17], and prosthetic foot design [18].

Rolling of a KS occurs when the ground contact and applied vertical force $\left(F_{v}(\theta)\right)$ are not aligned. Rolling KSs have been previously simulated in [1], presenting the kinematics of a plate being dispensed by a forced KS. However, this KS simulation was analyzed using a numerical approximation, while the exact solution to this KS dynamics problem was still 


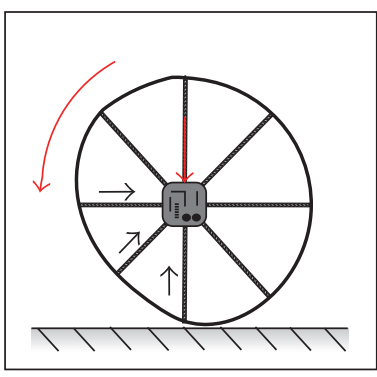

(a)

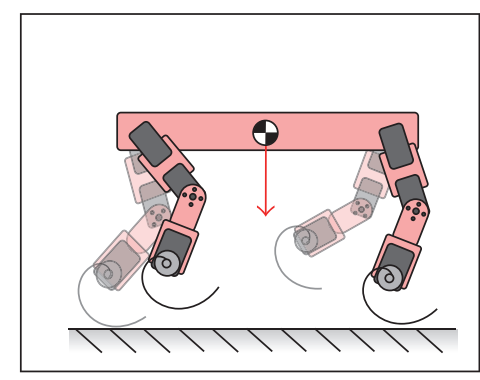

(b)

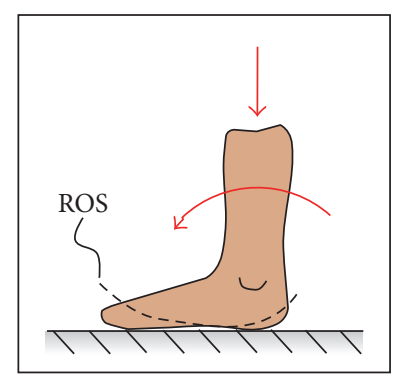

(c)

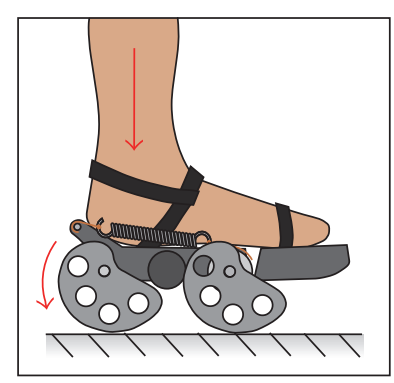

(d)

Figure 2: Examples of how different shapes are used to affect rotational and linear dynamics: (a) Two-Dimensional Crawling Deformable Soft Robot [6], (b) Quadruped with Resolute Spiral Feet [7], (c) foot roll-over shape (ROS), and (d) Gait Enhancing Mobile Shoe (GEMS) $[17,19]$.

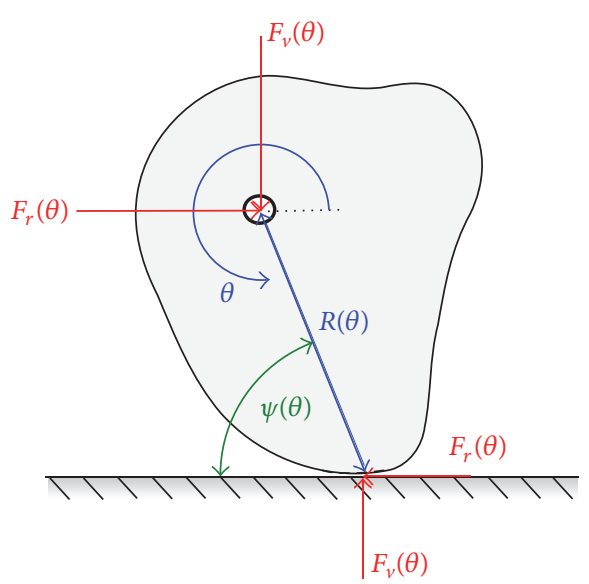

FIgURE 3: A physical kinetic shape curve $(R(\theta))$ in static equilibrium will react with a defined horizontal (parallel to the ground) force function $\left(F_{r}(\theta)\right)$ when a known vertical (perpendicular to the ground) force function $\left(F_{r}(\theta)\right)$ is applied to the curve's origin.

unsolved. In this article, we will be solving and verifying this exact setup with two different KSs.

\subsection{Rolling Shapes in Gait Rehabilitation and Assistance.} Rehabilitation engineering, specifically gait rehabilitation and foot prosthetics, can greatly benefit from an expanded understanding of the dynamics of different types of shaped curves rolling over ground. A foot roll-over shape (ROS), shown in Figure 2(c), is the curve that the foot rolls over while pivoting over the ground during the stance phase in walking. The form of a ROS is considered a vital concept in foot prosthesis design and greatly affects the balance and dynamics of normal or pathological gait $[18,20]$. The latest research on ROSs and prosthetic foot design lacks the ability to predict ROS behavior, while mentioning that an analytical solution to ROS rolling dynamics could be beneficial for improving the design of foot prostheses [21]. For instance, in prosthetic foot design, a properly adjusted ROS can moderate ground reaction forces channeled to a user's stump [22]. Recent studies mention that ROSs can be improved and modified by utilizing customized KSs to manipulate ground reaction forces [1].
A general solution to the forced dynamics of a KS can be utilized to improve an asymmetric gait rehabilitation device known as the Gait Enhancing Mobile Shoe (GEMS) [23, 24]. The GEMS is a shoe used to even out gait asymmetry of individuals that have suffered from a stroke. As the user steps onto a set of Archimedean spiral wheels, the wheels passively react by pushing the person's foot backwards (Figure 2(d)). This motion behaves similar to a split-belt treadmill that is commonly used in asymmetric gait correction $[25,26]$. With the use of KSs as wheels, such a device can be greatly improved by designing the wheel shape such that the GEMS produces predictable overground motions that can be customized to specific impairments. This article shows how to predict the resulting kinematics of the KSs that could potentially be used to define the GEMS wheels.

KSs could also benefit conventional crutches, which generally have a rubber point tip that the user swings/pivots over. Such a point tip mimics a constant radius (i.e., no radius change) when rolled over. Crutches do exist with a larger radial crutch tip; however these crutches have a large constant radius that does not change [27]. Point and constant radius tips cannot change the crutch walking dynamics. However, using a nonconstant crutch tip may alter crutch gait dynamics in a way that predictably assists or resists crutch movement during key positions and weight applications during the crutch gait cycle [28]. Such a nonconstant shape crutch tip can be customized to a variety of crutch walking styles, user disabilities, or energy expenditures. The exact motions of such a crutch tip shape can be predicted or customized with a greater understanding of KS rolling.

\section{Kinetic Shape Equation of Motion}

This section presents the derivation of a two-dimensional (2D) kinetic shape (KS) when it is not in static equilibrium. In particular, we derive the equations of motion that can predict the kinematics of a KS. Further this dynamic analysis is not exclusive to KSs but can predict the motions of any smooth and continuous physical curve that is known, measured, or approximated and is rolling across flat ground as a force is applied to the axle point.

As discussed previously in Section 2.1, a rotation moment is generated at the ground contact point when an arbitrary 


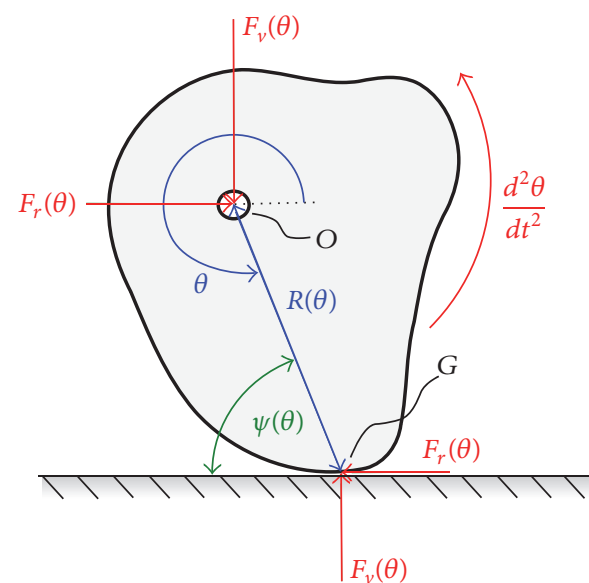

(a)

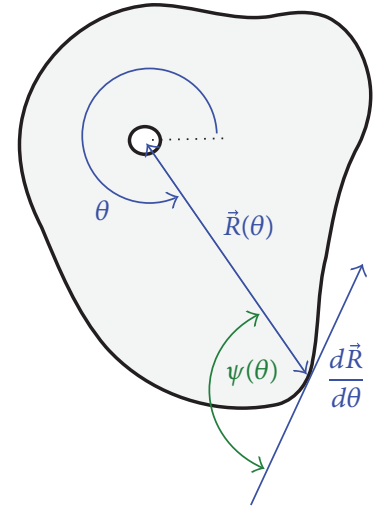

(b)

Figure 4: (a) A curved and continuous 2D object rolls as a force, $F_{v}(\theta)$, is applied to its axis. The applied force $\left(F_{v}(\theta)\right)$ is perpendicular to the flat rolling surface. (b) The polar tangential angle $(\psi(\theta))$ is measured between the shape radial and tangential vector.

smooth 2D shape is pressed onto a flat plane with a force directed through its axle point. We consider the case where this shape is not restrained and the ground moment is not matched, which results in the shape freely rotating about the ground contact point. This setup can be seen in Figure 4(a).

We assume that the friction force between the ground and the shape is large enough for the shape not to slip while rolling. It is also assumed that there is no deformation of the shape or ground. In a vertical setup, this analysis is valid when the applied force at the shape axle is much greater than the combined gravitational forces applied at the center of mass of the shape, or if the center of mass is concentrated at the shape axle. Note that in the forthcoming derivation, $R(\theta)$ is the $2 \mathrm{D}$ shape radius curve defined in polar coordinates and is a function of the angle $\theta$.

To derive the equation of motion for a $2 \mathrm{D} \mathrm{KS}$, we will begin by stating Isaac Newton's 2nd law [29] for general rotational rigid body dynamics in plane motion for a $2 \mathrm{D}$ rigid object rolling over ground

$$
\sum M=J_{G}(R, \theta) \frac{d^{2} \theta}{d t^{2}} .
$$

Here, $\sum M$ is the sum of all moments acting on the object, the polar mass moment of inertia of the shape about the ground contact point $(G)$ is defined as $J_{G}(R, \theta)$, and $d^{2} \theta / d t^{2}$ is the angular acceleration of the shape rolling over the ground contact point. As an arbitrary shape rolls, the polar moment of inertia changes with the shape orientation and with respect to the ground contact. Thus, it is important for us to formulate the polar mass moment of inertia such that it accounts for this reorientation. We start by defining (4) as the polar mass moment of inertia of an arbitrary 2D shape of variable thickness and heterogeneous material about the shape origin $(O),(R=0, \theta=0)$ :

$$
J_{O}(R, \theta)=\int_{A} R(\theta)^{2} \rho(R, \theta) t(R, \theta) d A .
$$

Here, $\rho(R, \theta)$ is the continuous density across the shape and $t(R, \theta)$ is the continuous thickness throughout the shape. The differential area, $d A$, of the shape can be defined by the infinitesimal change in radius, $d R$, and arc length, $R(\theta) d \theta$, yielding

$$
J_{O}(R, \theta)=\int_{\theta_{i}}^{\theta_{f}} \int_{R_{i}}^{R_{f}} R(\theta)^{2} \rho(R, \theta) t(R, \theta) R(\theta) d R d \theta .
$$

$\theta_{i}$ and $\theta_{f}$ are the initial and final angles of the shape definition, respectively, while $R_{i}$ and $R_{f}$ are the initial and final radii of shape radius function $R(\theta)$, respectively. The polar mass moment of inertia is defined with a variable thickness and heterogeneous shape density; however due to the later physical verification experiment and application, we will consider the $2 \mathrm{D}$ shape to be of uniform density and thickness. This consideration produces

$$
J_{O}(\theta)=\frac{\rho t}{4} \int_{\theta_{i}}^{\theta_{f}} R(\theta)^{4} d \theta .
$$

Equation (6) describes the polar mass moment of inertia of the 2D kinetic shape about its axle point for rotations. However, we aim to calculate the polar moment of inertia of the 2D KS around the ground contact point $(G)$ to also account for rolling over ground. Applying the parallel axis theorem shifts the rotation point from the shape origin $(O)$ to ground contact $(G)$ to obtain (7), the polar mass moment of inertia of the 2D KS about the ground contact point. Modifying (6) into (7) is only required if the shape is both rolling and translating over ground:

$$
J_{G}(\theta)=\frac{\rho t}{4} \int_{\theta_{i}}^{\theta_{f}} R(\theta)^{4} d \theta+m_{\mathrm{KS}} R(\theta)^{2} .
$$

Here, $m_{\mathrm{KS}}$ is the mass of the entire shape and $R(\theta)$ is the distance from shape axle to ground contact $(O-G)$. The mass 
of a $2 \mathrm{D}$ shape with a nonconstant density and thickness is defined by

$$
m_{\mathrm{KS}}=\int_{A} \rho(R, \theta) t(R, \theta) d A
$$

Following similar steps and assumptions for a constant shape thickness and density, redefining the differential area $d A$, and integrating through the radial direction, the KS mass is redefined as

$$
m_{\mathrm{KS}}=\frac{\rho t}{2} \int_{\theta_{1}}^{\theta_{2}} R(\theta)^{2} d \theta .
$$

This completes the definition for the polar mass moment of inertia of a rolling shape. The kinematics are derived by summing moments with respect to the shape ground contact (Figure 4). This is achieved by combining (3) and (7), resulting in

$$
F_{v}(\theta) R(\theta) \cos [\psi(\theta)]=J_{G}(\theta) \frac{d^{2} \theta}{d t^{2}} .
$$

Here, $F_{v}(\theta)$ is the force function applied at the shape axle $(O)$ and is perpendicular to the rolling surface. We define the polar tangential angle $(\psi(\theta))$ at an angular position $(\theta)$ between the polar curve vector $(\vec{R}(\theta))$ and tangential vector $(d \vec{R} / d \theta)$ in (11) and is shown in Figure 4(b):

$$
\psi(\theta)=\tan ^{-1}\left(\frac{R(\theta)}{d R / d \theta}\right) .
$$

Equation (10) can be rearranged to define the angular acceleration of the KS as a vertically applied force $\left(F_{v}(\theta)\right)$ is applied to its axle:

$$
\ddot{\theta}=\frac{d^{2} \theta}{d t^{2}}=\frac{F_{v}(\theta) R(\theta) \cos [\psi(\theta)]}{J_{G}(\theta)} .
$$

Equation (12) can be integrated to solve for angular velocity and position with respect to time, $t$. The constants of integration can be solved by applying initial angular velocity $\left(\dot{\theta}_{i}\right)$ and angular position $\left(\theta_{i}\right)$ of the KS at $t=0$. Equation (13) represents the angular velocity of the KS with time, while (14) defines the KS angular position:

$$
\begin{aligned}
& \dot{\theta}=\frac{d \theta}{d t}=t \frac{F_{v}(\theta) R(\theta) \cos [\psi(\theta)]}{J_{G}(\theta)}+\dot{\theta}_{i}, \\
& \theta=\theta(t)=t^{2} \frac{F_{v}(\theta) R(\theta) \cos [\psi(\theta)]}{2 J_{G}(\theta)}+t \dot{\theta}_{i}+\theta_{i} .
\end{aligned}
$$

Rearranging (10) again, we are able to predict the applied vertical force function $\left(F_{v}(\theta)\right)$ required to achieve a specified/known angular acceleration $\left(d^{2} \theta / d t^{2}\right)$ with a $2 \mathrm{D}$ shape curve $(R(\theta))$. This definition of the applied vertical force is shown in

$$
F_{v}(\theta)=\frac{J_{G}(\theta)\left(d^{2} \theta / d t^{2}\right)}{R(\theta) \cos [\psi(\theta)]} .
$$

It is also possible to redefine the sum of moments in (3) in terms of the radial ground reaction force (parallel to the ground) $\left(F_{r}(\theta)\right)$ instead of the applied vertical force $\left(F_{v}(\theta)\right)$. Note that the cosine trigonometric function in the numerator turns into a sine function

$$
\ddot{\theta}=\frac{d^{2} \theta}{d t^{2}}=\frac{F_{r}(\theta) R(\theta) \sin [\psi(\theta)]}{J_{G}(\theta)} .
$$

However, if both the applied force function perpendicular to ground $\left(F_{v}(\theta)\right)$ and the radially applied force function parallel to the ground $\left(F_{r}(\theta)\right)$ are known, (12) and (16) can be combined as

$$
\begin{aligned}
\ddot{\theta} & =\frac{d^{2} \theta}{d t^{2}} \\
& =\frac{R(\theta)\left\{F_{v}(\theta) \cos [\psi(\theta)]+F_{r}(\theta) \sin [\psi(\theta)]\right\}}{2 J_{G}(\theta)} .
\end{aligned}
$$

Note that solving (17) for the shape radius function $(R(\theta))$ would be very useful in that one could specify the vertically applied forces $\left(F_{v}(\theta)\right)$, the radial ground reaction forces $\left(F_{r}(\theta)\right)$, and a desired kinematics function over time $\left(d^{2} \theta / d t^{2}\right)$ that the resulting shape radius function $(R(\theta))$ will produce. However, if angular acceleration $\left(d^{2} \theta / d t^{2}\right)$ is considered to be a nonconstant, (10) yields a second order nonlinear partial differential equation dependent on angle $\theta$ and time $t$. Additionally, if we consider angular acceleration to be a constant, we are confronted with the problem of isolating and solving for the shape radius $(R(\theta))$ that is embedded in the trigonometric functions. Both assumptions could not be solved analytically; however they could be solved using numerical methods to find the shape curve radius function $(R(\theta))$.

\section{Physical Verification of Kinetic Shape Equation of Motion}

4.1. Modified Kinetic Shape Equation of Motion. To confirm our derived dynamic KS equations of motion ((12), (13), and (14)), we slightly modify them to match our physical experimental setup. This modification serves as a base for verification, while introducing an extension for practical applications of these dynamic KS equations.

The kinematics of two defined KSs were recorded with the experimental setup seen in the schematic depicted in Figure 5(a). Here, the KS does not roll over itself as it would over firm ground, but instead rolls about the rotation axle at the origin $(O)$ and over a flat and movable platform. As such, the $\mathrm{KS}$ rotates without translating, and the horizontal force applied by the KS causes the platform to move.

In this setup, it is not necessary to move the rotation point to ground contact with the parallel axis theorem as in (7), nor is it necessary to compute the total mass of the shape with (8). However, it is essential that the mass and travel direction of the dispensed platform is taken into account. Instantaneously, the platform moving underneath the KS can be modeled as a point mass attached at the shape-platform contact point $(P)$ and moving perfectly tangent to the KS curve. This is 


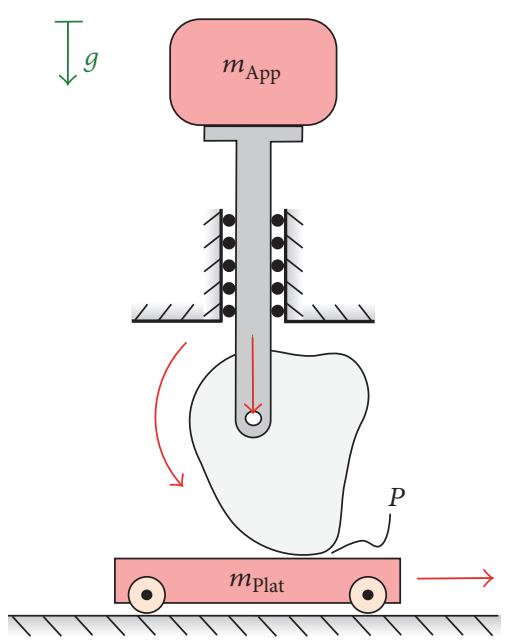

(a)

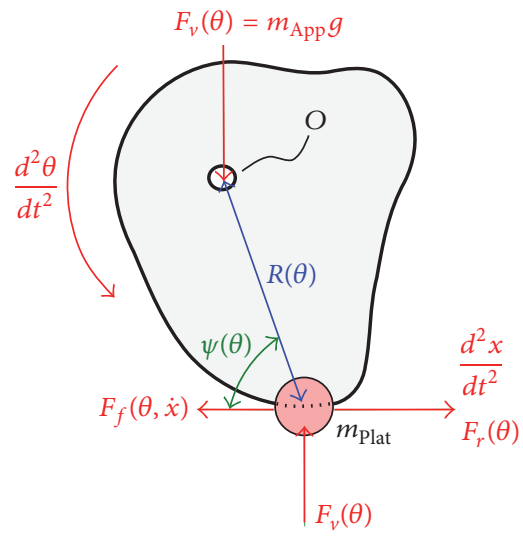

(b)

Figure 5: (a) Schematic of the setup used to verify the kinetic shape dynamics equations. A force that is perpendicular to the flat ground is applied to the shape origin with a known mass. As the shape rotates, it propels a platform $\left(m_{\text {plat }}\right)$ linearly. (b) Representation of the modeled parameters based on the experimental setup.

seen in Figure 5(b). The polar mass moment of inertia of that point mass at a radial distance $(R(\theta))$ forms the rotation point (origin) given in

$$
J_{\text {plat }}(\theta)=m_{\text {plat }}\{R(\theta) \sin [\psi(\theta)]\}^{2} .
$$

$m_{\text {plat }}$ represents the total mass of the dispensed platform. Note the additional $\sin [\psi(\theta)]$ term that accounts for the platform's mass moving parallel to the ground plane and not circularly about the shape origin. Combining the polar mass moment of inertia of the KS in (6) with (18) results in the total polar mass moment of inertia for a KS propelling a platform linearly as shown in

$$
\begin{aligned}
& J_{\text {O,plat }}(\theta) \\
& =\frac{\rho t}{4} \int_{\theta_{i}}^{\theta_{f}} R(\theta)^{4} d \theta+m_{\text {plat }}\{R(\theta) \sin [\psi(\theta)]\}^{2} .
\end{aligned}
$$

Some nonconservative forces such as friction in rolling or sliding between components are inevitable in physical mechanical systems such as our experimental setup. Friction forces in our experimental system are accounted for by defining a friction model that determines the translational friction between the dispensed platform and the ground. This translational friction is directly dependent on the applied vertical force, $F_{v}(\theta)=m_{\text {app }} g$, which is exerted by the KS onto the platform. It is also dependent on the velocity of the platform relative to the ground $(\dot{x})$. The friction model, which defines the coefficient of friction $(\mu)$, is described as the sum of the Stribeck, Coulomb, and viscous friction forces, shown in

$$
\mu(\dot{x})=\operatorname{signum}(\dot{x})\left[\mu_{k}+\left(\mu_{s}-\mu_{k}\right) e^{-C_{f}|\dot{x}|}\right]+\mu_{k} \dot{x} .
$$

In (20), the Coulomb friction, or kinetic friction, is defined by $\mu_{k}$, while the static friction is represented by $\mu_{s}$. The viscous friction is the product of the Coulomb term and the linear velocity $(\dot{x})$. The friction is dependent on the signum function of the linear velocity, which has possible values of -1 , 0 , and 1 . Finally, the $C_{f}$ term describes the exponential rate of the friction decay from static to kinematic. The horizontal friction force that the moving platform experiences due to friction is defined by

$$
F_{f}(\theta)=\mu(\dot{x}) m_{\mathrm{app}} g .
$$

Combining (17), (19), (20), and (21) defines the equation of motion of a platform $\left(m_{\text {plat }}\right)$ moving linearly due to the forces exerted by a $2 \mathrm{D} \mathrm{KS}$ :

$$
\begin{aligned}
\ddot{x}(\theta) & =\frac{d^{2} x}{d t^{2}} \\
= & \frac{F_{v}(\theta) R(\theta)[\cos [\psi(\theta)]-\mu(\dot{x}) \sin [\psi(\theta)]]}{J_{\text {O,plat }}(\theta)} \\
& \cdot R(\theta) \sin [\psi(\theta)] .
\end{aligned}
$$

Note the $R(\theta) \sin [\psi(\theta)]$ term is multiplied to isolate the acceleration component in the direction of the dispensed platform. Also note that in our setup $F_{v}(\theta)=m_{\text {app }} g$. Similar to the angular dynamics, linear velocity $(\dot{x}(\theta))$ and linear position $(x(\theta, t))$ of the platform can be derived by time integration and applying initial conditions, $\dot{x}_{i}$ and $x_{i}$. These equations are used to predict the linear motion of the dispensed platform as a derived $\mathrm{KS}$ is pressed on the platform.

However, in this particular setup, if the KS mass is negligible compared to the dispensed platform and the KS definition reaction radial function $\left(F_{r}(\theta)\right)$ and friction function $\left(F_{f}(\theta)\right)$ is known, it is also possible to predict the linear kinematics of the dispensed platform by the following simplified equation:

$$
\ddot{x}(\theta)=\frac{d^{2} x}{d t^{2}}=\frac{F_{r}(\theta)-F_{f}(\theta)}{m_{\text {plat }}}, \quad\left(m_{\text {plat }} \gg m_{\mathrm{KS}}\right) .
$$




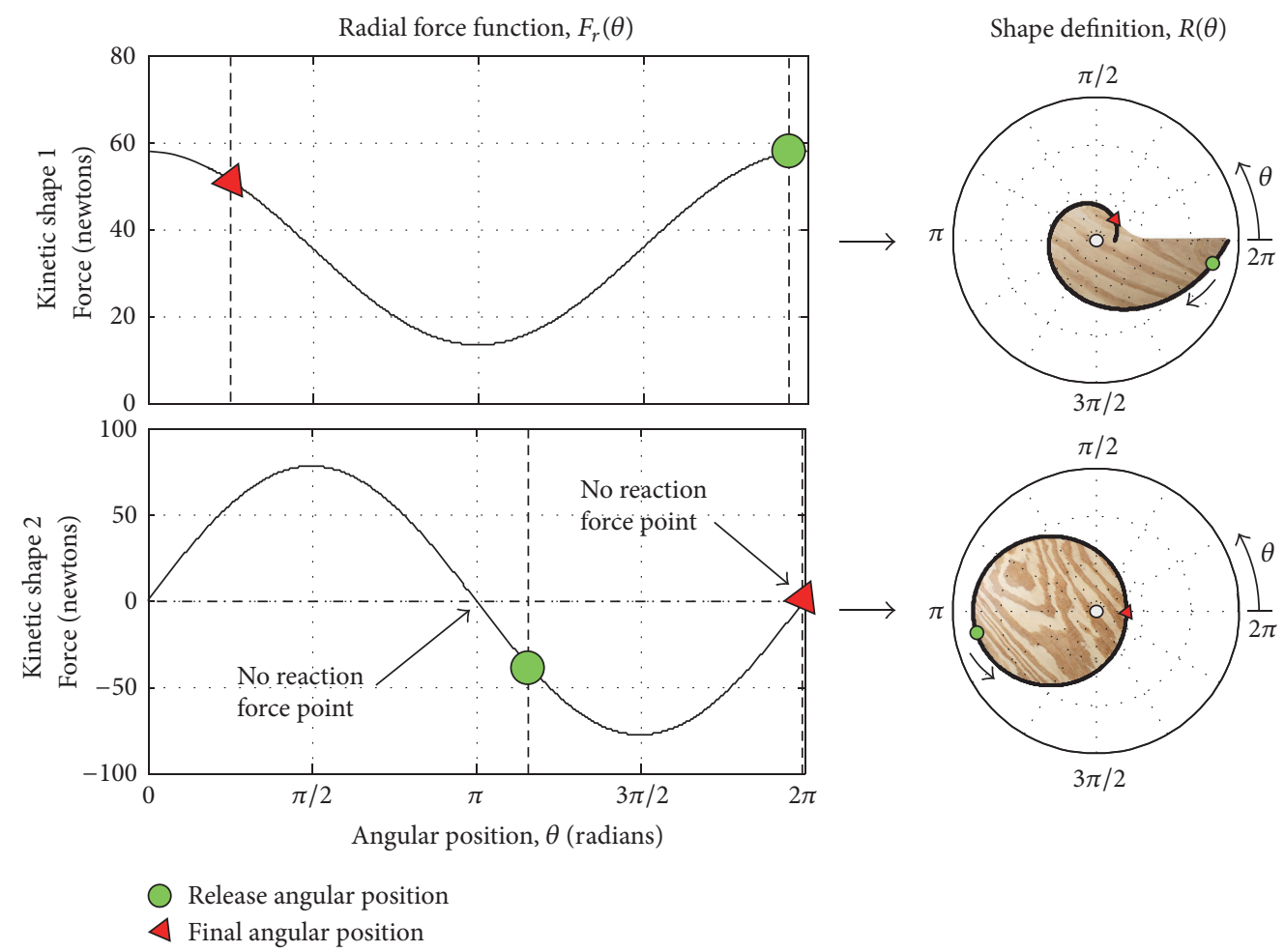

Figure 6: Two kinetic shapes selected for the verification measurement experiment. The applied vertical force function (perpendicular to ground), $F_{v}(\theta)=m_{\mathrm{app}} g$, and the radial force function (parallel to ground), $F_{r}(\theta)$, define the kinetic shape radius function, $R(\theta)$, in polar coordinates.

4.2. Experimental Kinetic Shape Definitions. Two distinctly different KSs are used for the evaluation of our derived equations of motion (22). Both shapes are defined by different desired radial ground reaction force functions $\left(F_{r}(\theta)\right)$ and a constant applied vertical weight, $F_{v}(\theta)=m_{\text {app }} g$. The magnitudes of the force functions were chosen to accommodate the experimental setup structure. The scale of the KSs was chosen to be relatively large to allow for a longer duration of shape movements, which makes it easier to observe its motions. Both KSs and their defining radial ground reaction forces ranged from 0 to $2 \pi$ radians and are shown in Figure 6.

The first KS is defined with a sinusoidal radial ground reaction force with a constant offset (24) and a constant applied weight function (25). Using the KS equation (Equation (2) [1]), the shape radius $(R(\theta))$ is found as (26):

$$
\begin{aligned}
& F_{r, 1}(\theta)=22.5 \cos (\theta)+35.6 \mathrm{~N}, \\
& F_{v, 1}(\theta)=120 \mathrm{~N}, \quad(27 \mathrm{lb} / 12.3 \mathrm{~kg}) \\
& R_{1}(\theta)=2.75 \exp [0.32 \theta+0.2 \sin (\theta)],
\end{aligned}
$$

$$
(0 \leq \theta \leq 2 \pi) .
$$

This first KS's radius ranges from $R_{1}(0)=2.75 \mathrm{in}(7.00 \mathrm{~cm})$ to $R_{1}(2 \pi)=20.51$ in $(52.1 \mathrm{~cm})$. The maximum physical dimension across this $\mathrm{KS}(\theta=0 \rightarrow \pi)$ is 28 in $71.2 \mathrm{~cm}$. The second shape and its force function are described in (28) and (27), yielding the KS radius function defined in (29):

$$
\begin{aligned}
& F_{r, 2}(\theta)=77.9 \sin (\theta) \mathrm{N}, \\
& F_{v, 2}(\theta)=120 \mathrm{~N}, \quad(27 \mathrm{lb} / 12.3 \mathrm{~kg}), \\
& R_{2}(\theta)=5.0 \exp [-0.71 \cos (\theta)], \quad(0 \leq \theta \leq 2 \pi) .
\end{aligned}
$$

This KS definition begins with an initial shape radius of $R_{2}(0)=5.00$ in $(12.7 \mathrm{~cm})$ and since the shape is defined by a sinusoidal function, it also ends with the same radius of $R_{2}(2 \pi)=5.00 \mathrm{in}(12.7 \mathrm{~cm})$. This continuity of the shape curve is due to its diminishing integral of its sinusoidal force definition and is explained further in [1]. The maximum physical dimension across this $\mathrm{KS}(\theta=0 \rightarrow \pi)$ is $25.3 \mathrm{in}$ $(64.2 \mathrm{~cm})$.

\subsection{Experimental Methods and Materials}

4.3.1. Fabrication. The physical device used to verify our linear kinematic equations is illustrated in Figure 7.

The shapes defined in the previous section were intentionally designed to be large in order to allow for ample recording time and resolution of their respective dynamics. Due to their relatively large size, the KSs were traced onto a pine wood panel (thickness, $t=1.59 \mathrm{~cm}$, density, $\rho=$ $633 \mathrm{~kg} / \mathrm{m}^{3}$ ) using a 1:1 scale printed stencil and subsequently 


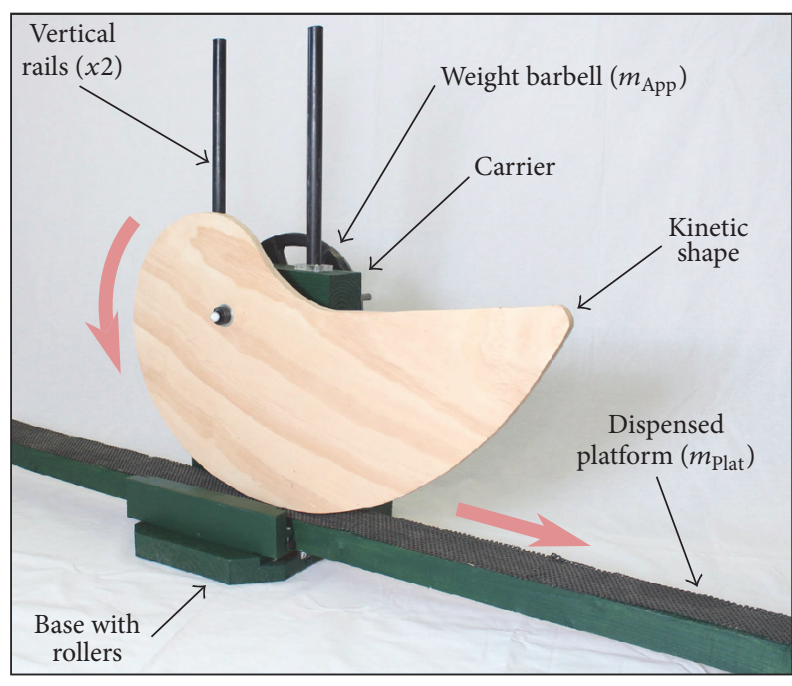

FIgURE 7: Actual setup used to measure the dynamics of the kinetic shape pushing a flat platform. A weight forces a defined kinetic shape onto a linearly moving platform, pushing the platform underneath itself.

cut out using a jigsaw. All rough edges were carefully sanded down to avoid irregularities around the shape perimeter.

The KS was attached at its origin to a carrier via a ball bearing. The ball bearing with the KS was carried by a 0.375 in $(0.95 \mathrm{~cm})$ steel rod axle that was pressed through the carrier. While the KS was attached on one side of the carrier, a weight barbell with a known weight was hung onto the other side of the steel rod axle. The two steel tube rails $(1.0$ in $(2.54 \mathrm{~cm})$ diameter) on which the carrier traveled vertically were firmly pressed into a fastened base platform. To ensure low friction between these two vertical rails and the carrier's nylon plastic sleeve bearings, the rails were lubricated with lithium grease. It is important to note that the entire carrier assembly itself weighs $2.0 \mathrm{lb}(0.91 \mathrm{~kg})$, which was included in the applied vertical force $\left(F_{v}(\theta)\right)$ for each KS definition.

The fastened base platform had one roller track directly beneath the KS that carried a moving platform $\left(m_{\text {plat }}\right)$. The platform traveling on the roller track is a 2 in $(5 \mathrm{~cm}) \times$ 3 in $(7.6 \mathrm{~cm}) \times 64$ in $(162 \mathrm{~cm})$ pine wood material weighing $m_{\text {plat }}=5.50 \mathrm{lb}(2.50 \mathrm{~kg})$. The track consisted of small rubber rollers below and on the sides of the platform for support. To maximize the friction (and reduce slippage) between the KS and the moving platform, thin rubber was stapled onto the surface of the dispensed platform.

Static friction $\left(\mu_{s}\right)$ and kinetic friction $\left(\mu_{k}\right)$ between the moving platform and the base roller track were measured by placing known and incrementing weights of $10 \mathrm{lb}(4.5 \mathrm{~kg})$, $20 \mathrm{lb}(9.0 \mathrm{lb})$, and $25 \mathrm{lb}(11.3 \mathrm{~kg})$ on top of the platform, while pulling one end of the platform with an analog fish scale (hanging scale). From these three applied weights, we approximated the average static and kinetic coefficients of friction using (21) and found them to be $\mu_{s}=0.350$ and $\mu_{k}=0.125$. We defined the exponential decay coefficient $\left(C_{f}\right)$ for the friction model to be 9.0 .
4.3.2. Motion Tracking. The analysis of the kinematics of both KSs was achieved via a Vicon ${ }^{\circledR}$ passive infrared camera marker motion tracking system, which included ten infrared cameras (Vicon Bonita B10 cameras) recording at $120 \mathrm{~Hz}$. The infrared markers that we used were $14 \mathrm{~mm}$ in diameter and were placed onto the front and back of the moving platform. The measured position data $(x(\theta))$ was numerically differentiated (finite difference formula) for linear velocity $(d x / d t)$ which in turn was filtered using a first order Butterworth low pass filter with a normalized cutoff frequency of $10 \mathrm{~Hz}$. The filtered velocity was then numerically differentiated for linear acceleration $\left(d^{2} x / d t^{2}\right)$. All postprocessing was completed in Matlab $^{\circledR} 2015 \mathrm{a}$.

4.3.3. Experimental Procedure. The linear dynamics of the moving platform was recorded for three trials for each KS (six trials total). The shapes were released at different angles as denoted in Figure 6. The first KS was placed onto the platform at an orientation of 6.1 radians $\left(345^{\circ}\right)$ as the weight was applied, while the second KS was positioned at an initial contact point angle of 4.9 radians $\left(280^{\circ}\right)$.

4.3.4. Simulation Model. In addition to the physical experiment, a Matlab script that simulated the physical experiment by applying our derived formulas was utilized to compare the modeled and measured results. The simulated results included linear acceleration, velocity, and displacement of the dispensed platform. The simulation was evaluated at a $d t=0.002 \mathrm{~s}$ time step and a $\theta=0.002$ rads angle step size around the KS definition.

4.4. Measured versus Simulated Dynamics Comparison. The measured experimental results and predicted simulation values for the linear displacement, velocity, and acceleration of the dispensed platform by both KSs are shown in Figure 8 .

The first KS rolled for 1.4 seconds to an angular position of $\pi / 4$ radians $\left(45^{\circ}\right)$. This motion moved the dispensed platform a distance of $1.1 \mathrm{~m}$ (measured) compared to the modeled distance of $0.9 \mathrm{~m}$. It stopped rolling as the end of the shape at $2 \pi$ radians hits the ground plane. The modeled displacement, velocity, and acceleration results were in close proximity and slightly lower to the recorded values. While not always aligned within one standard deviation of the recorded data, the simulated data always followed the same trend as the recorded data.

Notice that the linear acceleration of the pushed platform in the simulated model jumps to around $3 \mathrm{~m} / \mathrm{s}^{2}$, while the recorded data rises gradually to $2 \mathrm{~m} / \mathrm{s}^{2}$. This difference accounts for the overall shorter travel distance. This discrepancy can be explained by the friction model coefficients that were used. The friction model that was used assumes a high static friction (stiction) value after which it diminishes to an increasing viscous kinetic friction. Additionally, the exact and real frictional forces experienced during the short rolling period may be highly nonlinear and not easily modeled.

The second KS was recorded for 1.4 seconds, before the system came to a halt at an angular position of 0 radians (or $2 \pi$ radians). The linear position of the pushed platform oscillated around a specific position, which was when the KS 

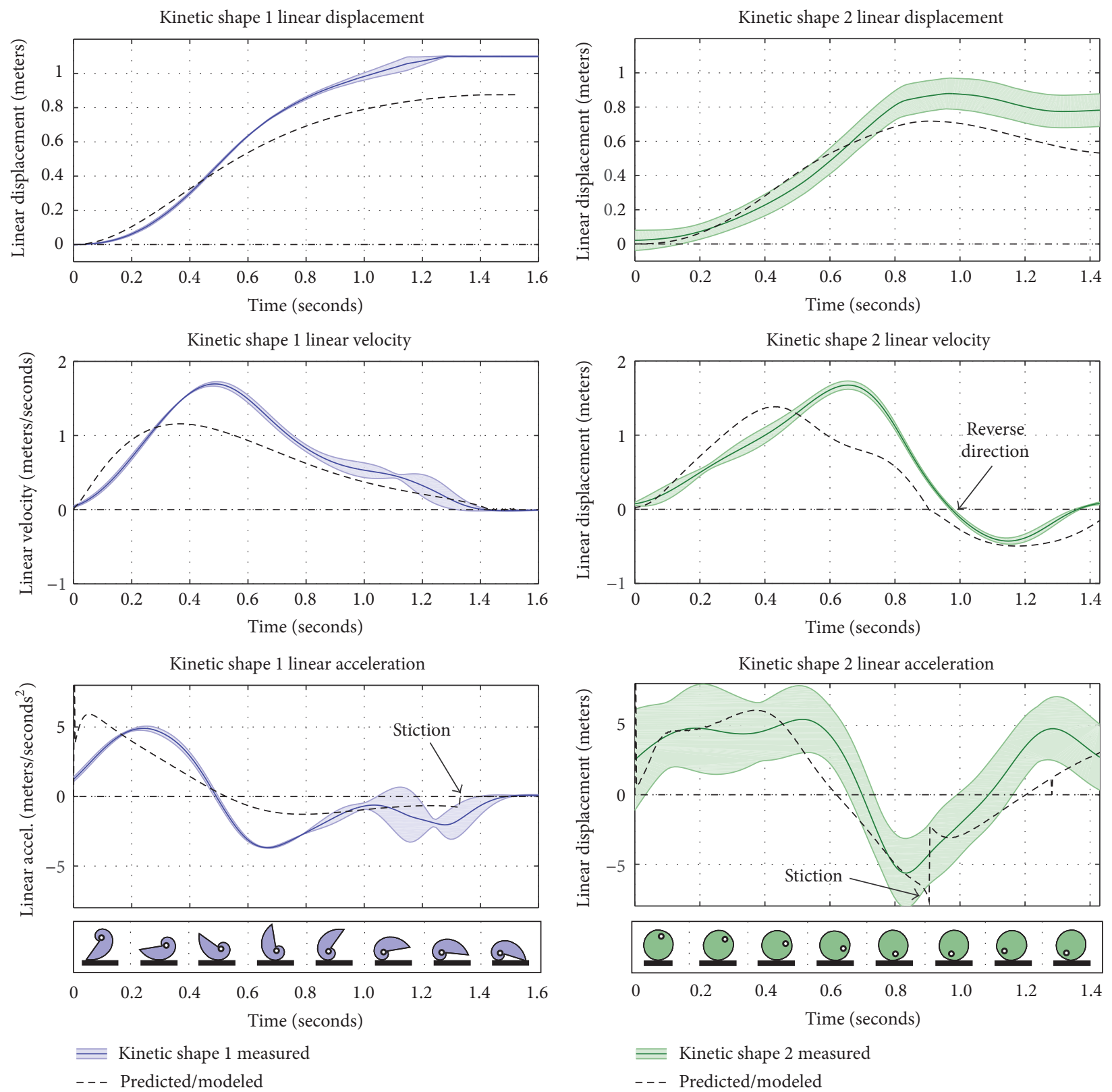

FIGURE 8: Kinetic shape dynamics measurement compared to the simulation. The displacement of the physical kinetic shape is measured and is integrated to determine the velocity and acceleration.

rolled around its 0 radians (or $2 \pi \mathrm{rad}$ ) angular position. At this equilibrium point, the radial reaction force $\left(F_{r}(\theta)\right)$ is a local minimum of the radial ground reaction force function (Figure 6). The platform at that equilibrium point was found to linearly move back and forth with a vibration amplitude of approximately $0.2 \mathrm{~m}$. This zero-force oscillation point is found where zero-acceleration is measured, which is at $0.68 \mathrm{~s}$ for the measured data and $0.6 \mathrm{~s}$ for the model. Similar to the first KS, the predicted values are in close proximity to the recorded values.

Notice that as the linear platform velocity crosses zero and the platform instantaneously comes to a halt at 0.9 seconds, the predicted model experiences an immediate increase in linear acceleration. This jump is due to the friction suddenly increasing until it reaches the stiction value (20). Although more damped and continuous, this stiction is also observed in the recorded acceleration data. This suggests that the friction model we have chosen for this application was too elementary in nature. The real and total friction affecting our apparatus is much more complex. Complexity in friction can be the result of imperfect alignments and movements throughout the dynamics, or nonlinear and complex cumulative friction characteristics throughout the entire system that may be difficult to model.

This experiment proved that our dynamic equations can be used to predict KS rolling kinematics while pushing a 


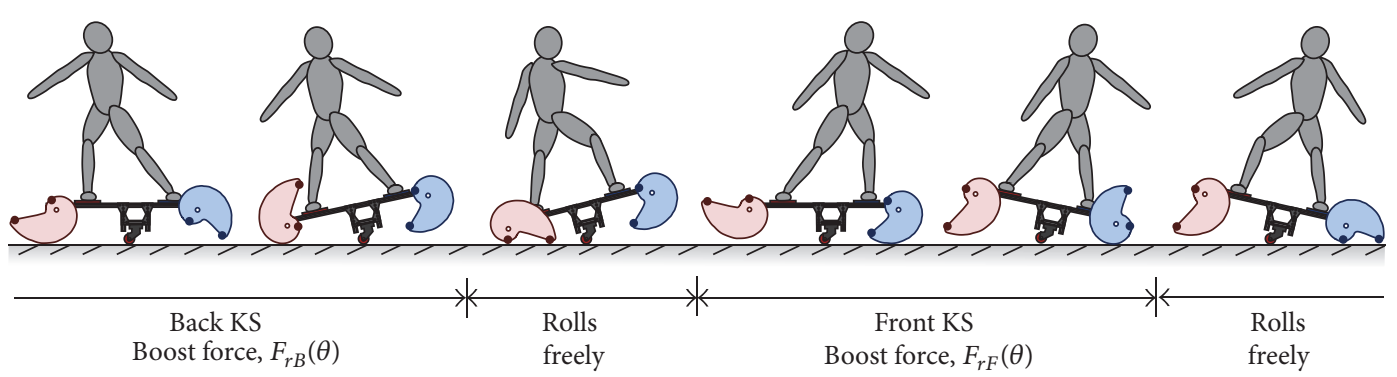

FIGURE 9: The motion of a kinetic board during usage. As the user loads the back kinetic shape with their weight, the kinetic shape reacts by rolling with a predefined and known horizontal ground reaction force. As the kinetic shape rolls, it flips onto two small rollers attached to the perimeter of the kinetic shape. The engagement of these rollers enables the angular kinetic shape rotation to be transferred into linear motion. The user subsequently pivots over a middle roller to load the front kinetic shape, which reacts with the same mechanism. By simply rocking back and forth, the user is able to continuously propel oneself forward.

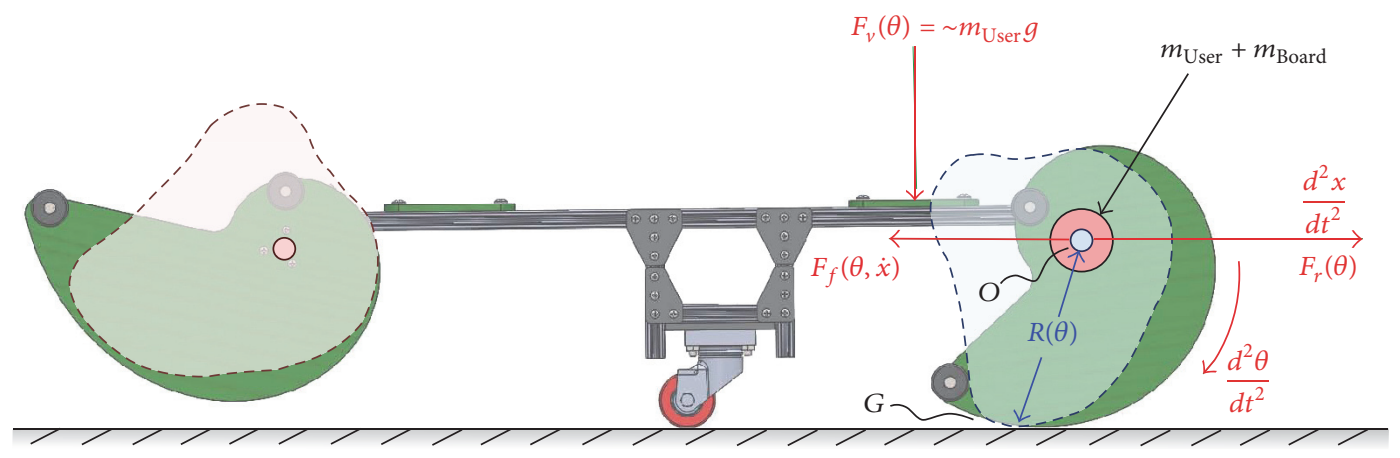

FIGURE 10: A schematic showing the kinetic board's kinetic shape wheels as a user applies their weight on the front stepping platform. The user's weight on the stepping platform is assumed to be perpendicular to the ground and transfered to the kinetic shape axle as $F_{v}(\theta)$. The kinetic board and the user are pushed by the kinetic shape axle. The user's mass $\left(m_{\text {user }}\right)$ and kinetic board mass $\left(m_{\text {board }}\right)$ are assumed to be concentrated at the kinetic shape axle.

platform out. This model can be applied for a KS or arbitrary continuous and irregular shapes for which the radial function $(R(\theta))$ is known, measured, or approximated. The experimental apparatus was not ideal and has room for an improved setup that is smoother and more aligned; however, the simulation was able to approximate actual values.

\section{The Kinetic Board for Transportation}

One novel application where our dynamic kinetic shape (KS) equations can be used is the kinetic board. Fundamentally, the kinetic board is a transport system that utilizes $\mathrm{KS}$ wheels and could be used by individuals to continuously propel themselves forward. As a user periodically shifts their weight while standing on the kinetic board, the board creates a predictable, smooth, and continuous linear forward overground movement. The kinetic board concept is depicted and outlined in Figure 9.

As seen in the figure, the kinetic board uses one KS in the back and one in the front. As the user applies their weight onto each shape, the KSs react by rolling, propelling the device and the user forward. Each KS has two small rollers at the shape perimeter and are positioned such that when the KS wheels roll around and collide with the ground, the two small rollers engage the ground and transfer the KSs' rotational motion into linear forward motion. The motion of each KS is dependent on its radius function $(R(\theta))$ and can be customized depending on the KS's defining position dependent force functions, $F_{v}(\theta)$ and $F_{r}(\theta)$.

5.1. Modified Kinetic Shape Equation of Motion. The equation of motion of linear forward movement of the kinetic board during its use is described by (22) in Section 4.1. In Section 4.1, a platform is dispensed linearly underneath a $\mathrm{KS}$ as the KS rolls on top of platform. In the case of the kinetic board, the shape rolls linearly over firm ground while pushing the board and the user's mass forward at the shape axle $(O)$ and parallel to the ground.

The schematic of the kinetic board as the user steps onto the front KS wheel is shown in Figure 10. In order to properly conform to this setup, we require an adjustment to the definition of the polar mass moment of inertia about the ground contact point $(G)$. This redefinition is defined by

$$
\begin{aligned}
J_{G}(\theta)= & J_{\mathrm{O}}(\theta)+m_{\mathrm{KS}} R(\theta)^{2} \\
& +\left(m_{\text {board }}+m_{\text {user }}\right)\{R(\theta) \sin [\psi(\theta)]\}^{2} .
\end{aligned}
$$




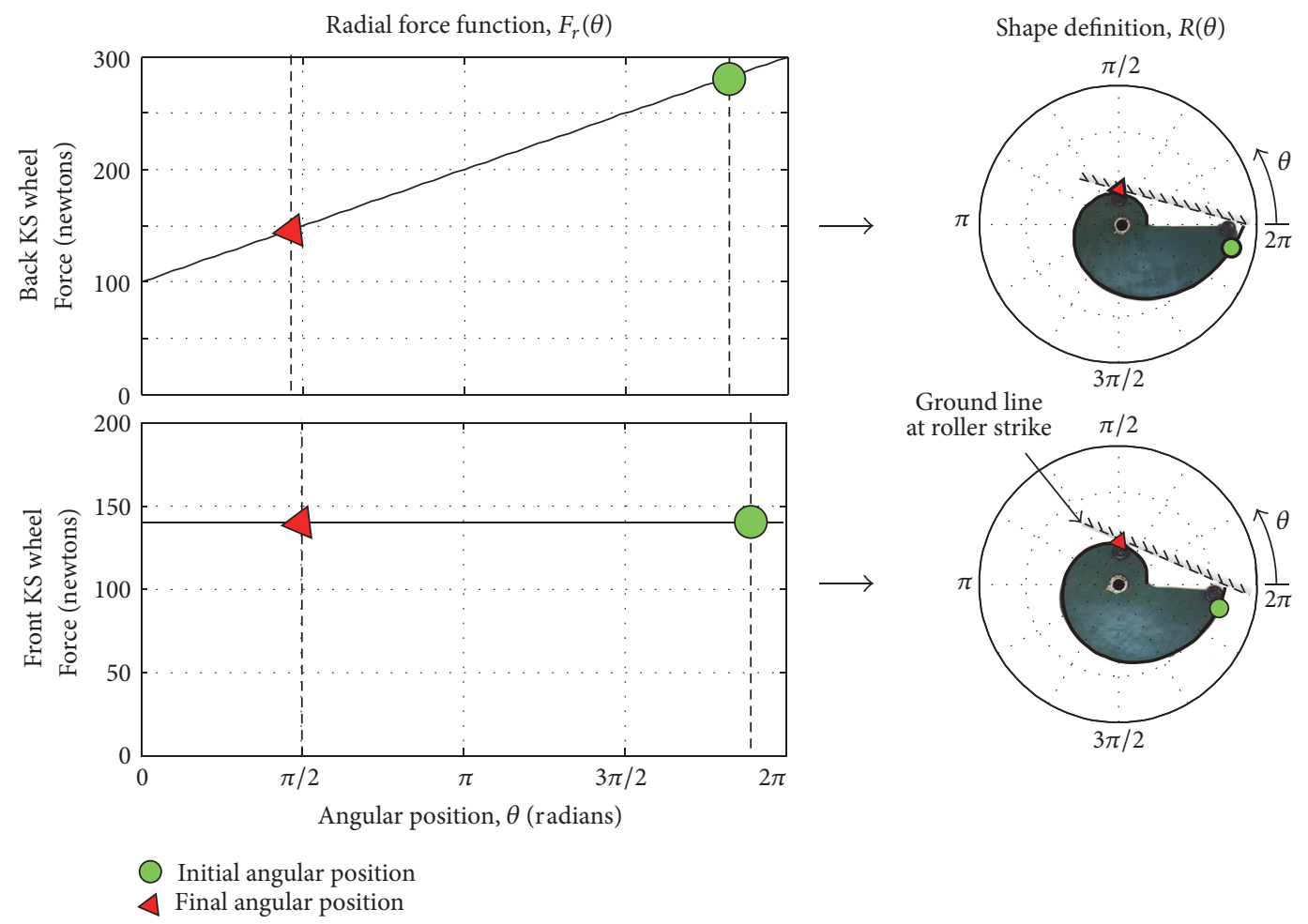

FIGURE 11: Kinetic shapes selected for the kinetic board back and front wheels. The definition of the kinetic shapes was selected to accommodate user applied forces.

Here, the first term, $J_{O}(\theta)$, is the polar mass moment of inertia of the $\mathrm{KS}$ around the axle point $(O)$ and is defined by (6) in Section 3. The middle term, $m_{\mathrm{KS}} R(\theta)^{2}$, transfers the rotation point from the shape axle $(O)$ to ground contact $(G)$ by the parallel axis theorem, where $m_{\mathrm{KS}}$ is the total mass of the kinetic shape (9). The last term, $\left(m_{\text {board }}+\right.$ $\left.m_{\text {user }}\right)\{R(\theta) \sin [\psi(\theta)]\}^{2}$, is a point mass at the KS axle point moving forward and into the direction parallel to the ground, where $m_{\text {board }}$ is the mass of the kinetic board and $m_{\text {user }}$ is the mass of the user riding on top of the kinetic board.

As before, (20) was utilized to develop a friction model in order to more accurately represent the derived results in comparison to the experimental. This friction model $(\mu(\dot{x}))$ aims to predict the total friction experienced by the kinetic board when moving forward.

5.2. Kinetic Shape Definitions. With the consideration of the analysis of a preliminary kinetic board prototype, the parameters of both KS wheels of the kinetic board were strategically selected. Design criteria for the wheels were to allow for adequate and smooth forward motion without drastically accelerating the board. The definitions of both back and front KS wheels for the kinetic board are shown in Figure 11.

As the user shifts their weight onto the KS wheels, the force applied by the user onto the wheels is not perfectly perpendicular to the ground but also includes a force component that is slightly parallel to the ground. This horizontal push force is down and to the rear of the board as the user loads the back KS wheel, which slightly resists the forward motion. This impedance only seemed present at the beginning of the rolling motion. Once the equilibrium is unbalanced and the kinetic board's inertia increased, the rear shape had no trouble rolling on and propelling the user forward.

Therefore, as the user leans onto the back KS wheel with user weight $F_{v B}(\theta)=m_{\text {user }} g$, we define its horizontal KS push force $\left(F_{r B}(\theta)\right)$ to initially push with a higher force while gradually decreasing as the KS wheel rolls (Figure 11). The vertically applied force $\left(F_{v B}(\theta)\right)$ by the user was chosen to be the weight of the measured user of the board which was $180 \mathrm{lb}(800 \mathrm{~N})(31)$. The horizontal reaction force $\left(F_{r B}(\theta)\right)$ is described by (32). The resulting radial shape function $\left(R_{B}(\theta)\right)$ is shown in (33). The initial radius, $R_{B}\left(\theta_{i}=0\right)$, for the back KS wheel was chosen to be 3.0 in $(0.077 \mathrm{~m})$ :

$$
\begin{aligned}
& F_{v B}(\theta)=800 \mathrm{~N}, \\
& F_{r B}(\theta)=\frac{200}{2 \pi} \theta+100 \mathrm{~N}, \\
& R_{B}(\theta)=0.077 \exp \left[\frac{\theta^{2} / 2+\pi \theta}{8 \pi}\right] \mathrm{m}, \quad(0 \leq \theta \leq 2 \pi) .
\end{aligned}
$$

As the user loads the front, the horizontal push force that is applied down and to the front of the board KS wheel assists the forward motion. In this case, we define the forward KS wheel horizontal push force to be constant (35). The resulting 


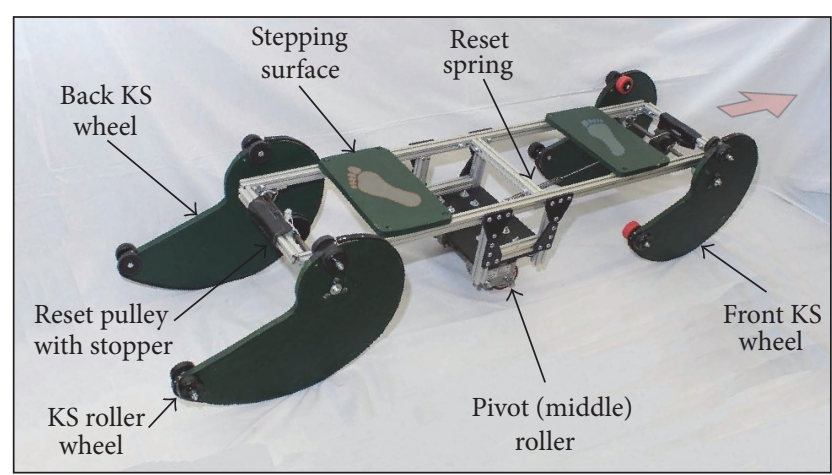

FIGURE 12: The actual kinetic board used to compare physical kinetic board measured and modeled dynamics.

radial shape function $\left(R_{F}(\theta)\right)$ is shown in (35) with a starting radius of 3.0 in $(0.077 \mathrm{~m})$ as well

$$
\begin{aligned}
& F_{v F}(\theta)=800 \mathrm{~N}, \\
& F_{r F}(\theta)=140 \mathrm{~N}, \\
& R_{F}(\theta)=0.077 \exp \left[\frac{7 \theta}{40}\right] \mathrm{m}, \quad(0 \leq \theta \leq 2 \pi) .
\end{aligned}
$$

\subsection{Experimental Methods and Materials}

5.3.1. Fabrication. The actual kinetic board used in this experiment is shown in Figure 12. The kinetic board is framed and held together by adjustable and rigid 1 in by 1 in $(2.54 \mathrm{~cm}$ by $2.54 \mathrm{~cm}$ ) $80 / 20^{\circledR}$ aluminum extrusion pieces. Two heightadjustable spring-loaded caster wheels with rubber rollers were used for the middle pivot.

Two 7 in by 12 in $(17.9 \mathrm{~cm}$ by $30.7 \mathrm{~cm})$ stepping platforms made of $0.625 \mathrm{in}(1.60 \mathrm{~cm})$ thick wood were attached on top of the frame fixed 25 in $(64 \mathrm{~cm})$ apart (center to center), which is a slightly greater distance than the average adult male's biacromial diameter (shoulder width) of 18 in $(46 \mathrm{~cm}$ ) [30].

The design included two sets of KS wheels on either side that are reset at each pivot of the kinetic board. A pretensioned extension spring with a stiffness of $2.37 \mathrm{lb} / \mathrm{in}(4.12 \mathrm{~N} / \mathrm{cm})$ resets the back and front set of KS wheels to their initial position. The springs were attached to a laser cut plastic pulley $\left(0.5\right.$ in $(1.27 \mathrm{~cm})$ thick $\times 3.0$ in $(7.62 \mathrm{~cm})$ diameter Delrin $\left.{ }^{\circledR}\right)$ that was attached to the KS wheel axle. The pulley was attached to the steel wheel axles. A quick-recovery natural gum foam stopper halted the KS wheel rotation as it was resetting.

The relatively large KS wheels on either side of the kinetic board were fabricated with the same material and technique outlined in Section 4.3. To ensure that the kinetic board could be used on various surfaces with a minimal amount of slip and a maximum ground-wheel friction, a strip of 0.25 in $(0.64 \mathrm{~cm})$ thick rubber $(60 \mathrm{~A}$ durometer) was firmly screwed (with countersink) to the perimeter the KS wheels. The kinetic shape wheels were attached to a $0.5 \mathrm{in}(1.27 \mathrm{~cm})$ steel shaft. The kinetic shapes were attached to the steel shaft axle via a keyed steel bushing. The steel shaft axle rotated smoothly on base mount ball bearings (two per shaft) attached to the bottom of the aluminum framing of the board.

The small rollers on the perimeter of the KS wheels were 0.5 in (1.28 in) rubber caster wheels with a diameter of 2.0 in $(5.12 \mathrm{~cm})$. For more stable rolling and to eliminate twisting moments, a 3 in $(7.68 \mathrm{~cm})$ shoulder bolt joined two roller wheels. Each entire wheel with steel bushing and four rubber rollers weighed approximately $m_{\mathrm{KS}}=3.2 \mathrm{lb}(1.45 \mathrm{~kg})$. By pulling the kinetic board with a hanging fish scale, the static friction $\left(\mu_{s}\right)$ was measured to be approximately 0.4 , while the kinetic friction $\left(\mu_{k}\right)$ was measured to be approximately 0.07 . The exponential decay coefficient $\left(C_{f}\right)$ was approximated to be 7.0.

The entire kinetic board design was approximately 48 in $(123 \mathrm{~cm})$ long, 17.6 in $(45 \mathrm{~cm})$ wide, and 12 in $(30.7 \mathrm{~cm})$ high (from ground) and had a total weight of around $m_{\mathrm{board}}=35 \mathrm{lb}$ $(15 \mathrm{~kg})$.

5.3.2. Motion Tracking. The same motion capture system and postprocessing process was used as described in Section 4.3. The linear overground position of the board as a whole $(x(t))$ was measured by placing a passive retroreflective infrared marker at the center of the middle pivot roller.

\subsubsection{User Applied Force Recording via Nintendo Wii Balance} Board. Since the KS wheel kinematics greatly depend on the forces applied to the KS wheels, it was necessary to dynamically measure how much force is applied to the back and front KS wheels as the kinetic board pivots back and forth. This measurement was achieved via two Nintendo Wii Balance Boards (WBB), one placed at each end of the kinetic board (Figure 13). This setup allows the board to be used, while wirelessly (Bluetooth 4.0) recording the forces $\left(F_{v}(\theta)\right.$ ) the user applies to each KS wheel.

A WBB is a plastic board $(51 \mathrm{~cm} \times 32 \mathrm{~cm} \times 5.3 \mathrm{~cm})$ that is primarily used in the gaming industry but has recently been utilized as a reliable force measurement device $[31,32]$ that is comparable to high end laboratory force analysis equipment [33]. The WBB is instrumented with four force sensors, one at each corner that allow for the measurement of a cumulative applied weight sampled at $50 \mathrm{~Hz}$ with a resolution of $\pm 5 \mathrm{~N}$. However, it is important to note that the WBB is only able to read forces that are orthogonal to the WBB stepping surface and not along the surface. The measured kinetics from the WBB were applied to the KS dynamics simulation model to predict the resulting kinematics.

5.3.4. Experimental Protocol. One healthy individual (age 23, weight $180 \mathrm{lb}(82 \mathrm{~kg})$, height 70 in $(178 \mathrm{~cm}))$ was recorded using the kinetic board three times. For each trial, after rocking back and forth two times, the kinetic board's position and user applied forces were recorded as the participant loaded the back KS wheel and then the front KS wheel.

5.4. Measured versus Simulated Dynamics Comparison. We only simulated the time periods when the KS wheel action was present, not during the time the board was coasting with the smaller rollers. Measured kinetic board kinematics are 

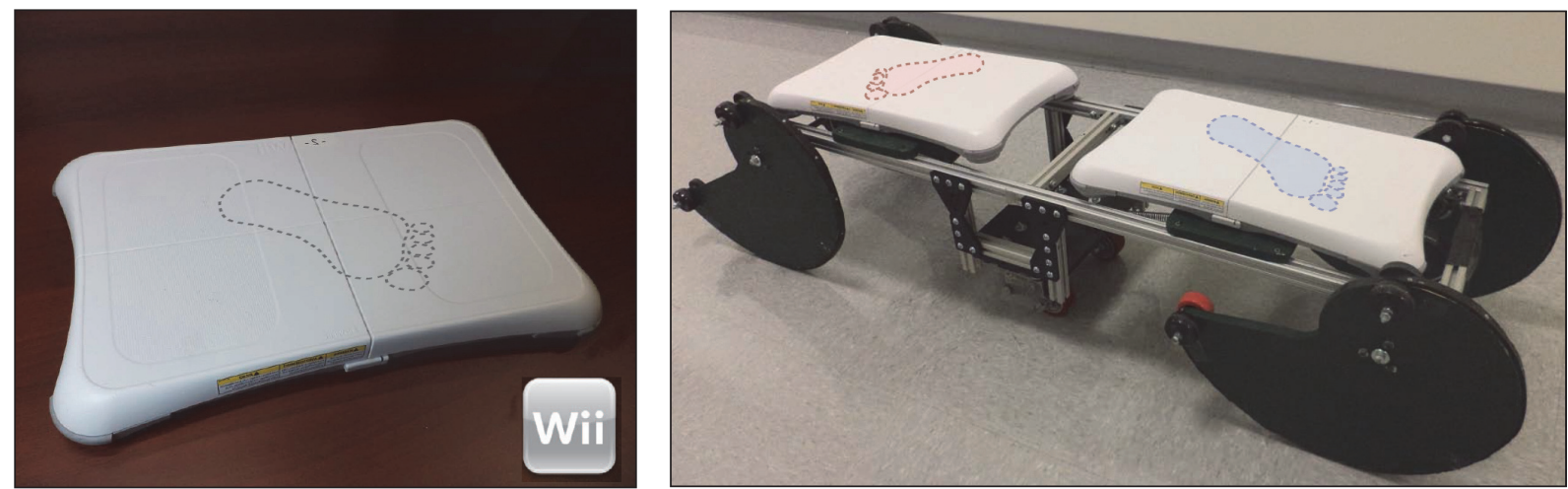

FIGURE 13: The force application by the kinetic board user onto the kinetic shape wheels was measured using two Nintendo ${ }^{\circledR}$ Wii ${ }^{\circledR}$ boards (WBB), one for the front foot and one for the rear. The portable and wireless WBB were only able to measure forces orthogonal to the stepping surface.

generally in agreement with the expected simulation data (Figure 14).

The kinematic motion capture data shows an expected trend as the kinetic board accelerates during KS engagement periods, while it decelerates during the cruising periods when the KS wheel flips over and rolls on its rollers. The acceleration is noticeably lower when the subject presses down on the front kinetic shape than the rear. Given an approximately equal magnitude of applied force by the user on the back and front KS wheels, we can conclude that the additional acceleration force is due to the force definition of the back KS wheel or discrepancy of kinetic board and user mass and applied forces.

The simulation model of the dynamic KS on the kinetic board is a good predictor of measured kinetic board movement. A bump in linear acceleration of the board during the initial front KS wheel engagement $(2.6 \mathrm{~s}-3.0 \mathrm{~s})$ was due to the subject slightly pressing forward and down onto the front KS. This horizontal force component could not have been recorded by the WBB; however it was noticeable in the kinematic video review.

The constant offset between the simulated and measured data may be due to the user consistently pushing backward or forward with their legs instead of simply leaning directly on top of the KS wheels. Note that the WBB used to record the input forces was only able to record forces orthogonal to the WBB stepping surface.

\section{Conclusion and Future Work}

We have derived the equations of motion for two-dimensional kinetic shapes (KSs) (or any other smooth curves) that can define the shape's angular and linear kinematics as a force is applied to the its axle/origin. As shown, this derivation can be adapted to a variety of uneven shape dynamics situations during force application.

We slightly altered these equations of motion to compare modeled dynamics with the dynamics of a matching physical experiment. In order to accurately model our physical setup,

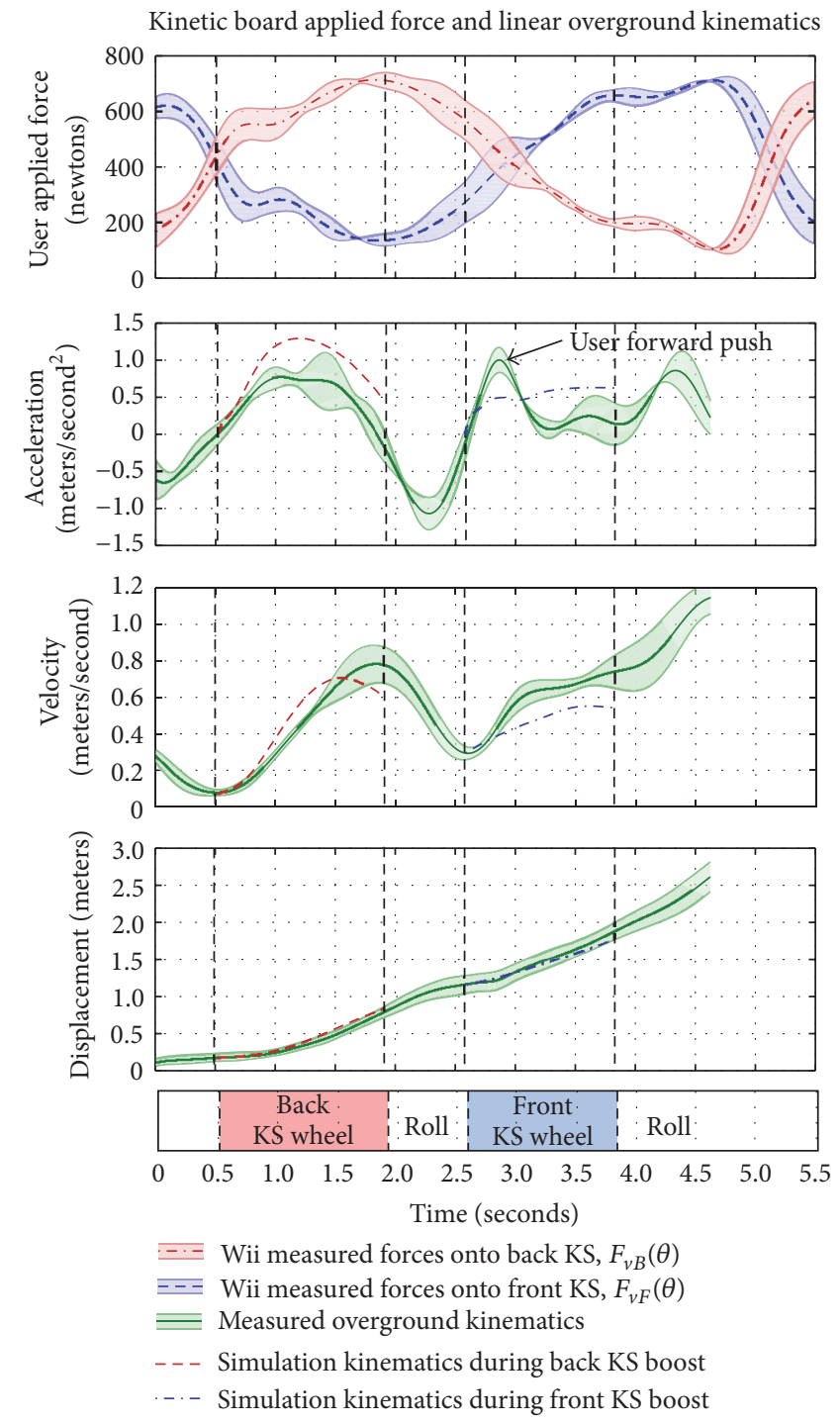

FIgURE 14: Kinetic board measured and simulated dynamics. The overground displacement is measured and is integrated to determine the velocity and acceleration. 
nonconservative friction forces were included into our KS dynamics equations. Although the general and resulting kinematics of the simulation of two tested KSs were in agreement with physical measurements, some differences between the expected and physical models were present; these can be reduced with a more accurate friction model definition and physical setup fabrication precision.

The dynamic KS equations were modified and applied to a novel transportation device called the kinetic board to successfully predict its motion. The comparison of simulated and measured kinetic board kinematics shows that the derived dynamic equations can be used to approximate the kinematics of the kinetic board. However, more fitting simulation results can be created with better modeling and measuring of user input forces.

Future works include further modifying the KS equations of motion to be able to derive exact shapes that produce desirable motions for specific applications such as the kinetic board, Gait Enhancing Mobile Shoe (GEMS) for stroke rehabilitation [17, 34], or an enhanced walking crutch tip [28]. The GEMS wheels could be designed such that they produce overground foot motions that are customized to particular individuals, walking asymmetries, or gait cycle instances. A tailored walking crutch tip could allow for easier maneuvering, which can potentially diminish overall fatigue from daily use, and allow us to tailor the dynamics of different crutch gait styles and walking environments.

\section{Competing Interests}

The authors declare that there is no conflict of interest regarding the publication of this paper.

\section{References}

[1] I. Handžić and K. B. Reed, "Kinetic shapes: analysis, verification, and applications," ASME Journal of Mechanical Design, vol. 136, no. 6, Article ID 061005, pp. 1-8, 2014.

[2] Archimedes, On Spirals, 225 B.C., http://www-history.mcs.stand.ac.uk/Curves/Spiral.html.

[3] F. Y. Chen, Mechanics and Design of Cam Mechanisms, Pergamon Press, New York, NY, USA, 18th edition, 1982.

[4] J. Gilchrist, J. Campbell, C. Donnelly, J. Peeler, and J. Delaney, "Spiral plate method for bacterial determination," American Society for Microbiology, vol. 25, no. 2, pp. 244-252, 1973.

[5] H. Sakata and M. Okuda, "Fluid compressing device having coaxial spiral members," US Patent 5,603,614, 1994.

[6] Y. Sugiyama and S. Hirai, Crawling and Jumping by a Deformable Robot, Springer, Berlin, Germany, 2006.

[7] A. Mutka, E. Koco, and Z. Kovacic, "Adaptive control of quadruped locomotion through variable compliance of revolute spiral feet," International Journal of Advanced Robotic Systems, vol. 11, 2014.

[8] G. Figliolini, P. Rea, and J. Angeles, "The pure-rolling camequivalent of the Geneva mechanism," Mechanism and Machine Theory, vol. 41, no. 11, pp. 1320-1335, 2006.

[9] S. P. Radzevich, Dudley's Handbook of Practical Gear Design and Manufacture, CRC Press, New York, NY, USA, 2012.

[10] J. E. Shigley, Shigley's Mechanical Engineering Design, vol. 8, McGraw-Hill, New York, NY, USA, 2008.
[11] E. Zheng, F. Jia, H. Sha, and S. Wang, "Non-circular belt transmission design of mechanical press," Mechanism and Machine Theory, vol. 57, pp. 126-138, 2012.

[12] E. Ottaviano, D. Mundo, G. A. Danieli, and M. Ceccarelli, "Numerical and experimental analysis of non-circular gears and cam-follower systems as function generators," Mechanism and Machine Theory, vol. 43, no. 8, pp. 996-1008, 2008.

[13] L. Hall and S. Wagon, "Roads and wheels," Mathematics Magazine, vol. 65, no. 5, pp. 283-301, 1992.

[14] J. Bloom and L. Whitt, "The geometry of rolling curves," The American Mathematical Monthly, vol. 88, no. 6, pp. 420-426, 1981.

[15] I. Handzic, Analysis and application of passive gait rehabilitation methods [Ph.D. dissertation], University of South Florida, 2014.

[16] I. Handžić and K. B. Reed, “The musical kinetic shape: a variable tension string instrument," Applied Acoustics, vol. 85, pp. 143149, 2014.

[17] I. Handžić, E. M. Barno, E. V. Vasudevan, and K. B. Reed, "Design and pilot study of a gait enhancing mobile shoe," Paladyn, Journal of Behavioral Robotics, vol. 2, no. 4, pp. 193201, 2011.

[18] C. Curtze, A. L. Hof, H. G. van Keeken, J. P. K. Halbertsma, K. Postema, and B. Otten, "Comparative roll-over analysis of prosthetic feet," Journal of Biomechanics, vol. 42, no. 11, pp. 17461753, 2009.

[19] K. B. Reed and I. Handžić, "Gait-altering shoes," U.S. Patent 9,295,302, March 2016.

[20] A. H. Hansen, D. S. Childress, and E. H. Knox, "Prosthetic foot roll-over shapes with implications for alignment of trans-tibial prostheses," Prosthetics and Orthotics International, vol. 24, no. 3, pp. 205-215, 2000.

[21] A. H. Hansen and C. C. Wang, "Effective rocker shapes used by able-bodied persons for walking and fore-aft swaying: implications for design of ankle-foot prostheses," Gait and Posture, vol. 32, no. 2, pp. 181-184, 2010.

[22] J. S. Rietman, K. Postema, and J. H. B. Geertzen, "Gait analysis in prosthetics: opinions, ideas and conclusions," Prosthetics and Orthotics International, vol. 26, no. 1, pp. 50-57, 2002.

[23] I. Handžić and K. B. Reed, "Motion controlled gait enhancing mobile shoe for rehabilitation," in Proceedings of the IEEE International Conference on Rehabilitation Robotics (ICORR '11), pp. 583-588, July 2011.

[24] I. Handžić, E. Vasudevan, and K. B. Reed, "Developing a gait enhancing mobile shoe to alter over-ground walking coordination," in Proceedings of the IEEE International Conference on Robotics and Automation (ICRA '12), pp. 4142-4129, May 2012.

[25] D. S. Reisman, H. McLean, J. Keller, K. A. Danks, and A. J. Bastian, "Repeated split-belt treadmill training improves poststroke step length asymmetry," Neurorehabilitation \& Neural Repair, vol. 27, no. 5, pp. 460-468, 2013.

[26] I. Handžić and K. B. Reed, "Comparison of the passive dynamics of walking on ground, tied-belt and split-belt treadmills, and via the Gait Enhancing Mobile Shoe (GEMS)," in Proceedings of the IEEE 13th International Conference on Rehabilitation Robotics (ICORR '13), pp. 1-6, IEEE, Seattle, Wash, USA, June 2013.

[27] M. A. Le Blanc, L. E. Carlson, and T. Nauenberg, "A quantitative comparison of four experimental axillary crutches," Journal of Prosthetics and Orthotics, vol. 5, no. 1, pp. 40-48, 1993.

[28] D. Capecci, S. H. Kim, K. B. Reed, and I. Handzic, "Crutch tip for swing-through crutch walking control based on a kinetic shape," in Proceedings of the 14th IEEE/RAS-EMBS International 
Conference on Rehabilitation Robotics (ICORR '15), pp. 612-617, Singapore, August 2015.

[29] I. Newton, F. Cajori, R. T. Crawford, and A. Motte, Sir Isaac Newton's Mathematical Principles of Natural Philosophy and His System of the World, Edited by R. T. Crawford, University of California Press, Translated by M. Andrew in 1729, The Translations Revised and Supplied with an Historical and Explanatory Appendix by Florian Cajori, 1934.

[30] H. W. Stoudt, A. Damon, R. McFarland et al., Skinfolds, Body Girths, Biacromial Diameter, and Selected Anthropometric Indices of Adults, United States, 1960-1962, US Health Services and Mental Health Administration, 1970.

[31] D. J. Goble, B. L. Cone, and B. W. Fling, "Using the Wii Fit as a tool for balance assessment and neurorehabilitation: the first half decade of 'wii-search", Journal of NeuroEngineering and Rehabilitation, vol. 11, no. 1, article 12, 2014.

[32] I. Handžić and K. B. Reed, "Analysis of human stepping dynamics using a Wii balance board with a webcam: a comparison study," in Proceedings of the 8th ACM International Conference on Pervasive Technologies Related to Assistive Environments, p. 52, Curfu, Greece, July 2015.

[33] A. Huurnink, D. P. Fransz, I. Kingma, and J. H. van Dieën, "Comparison of a laboratory grade force platform with a Nintendo Wii Balance Board on measurement of postural control in single-leg stance balance tasks," Journal of Biomechanics, vol. 46, no. 7, pp. 1392-1395, 2013.

[34] S. H. Kim, I. Handžić, D. Huizenga et al., "Initial results of the gait enhancing mobile shoe on individuals with stroke," in Proceedings of the International Conference of the IEEE Engineering in Medicine and Biology Society (EMBC '16), Orlando, Fla, USA, 2016. 


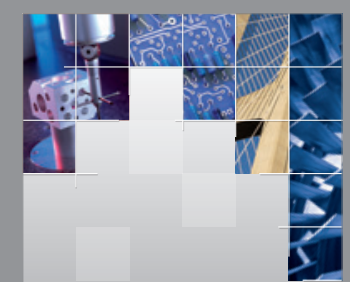

\section{Enfincering}
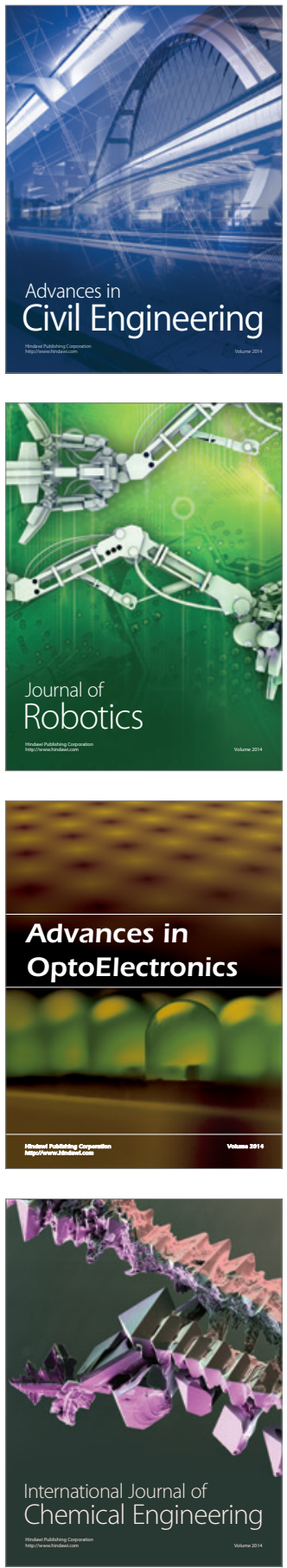

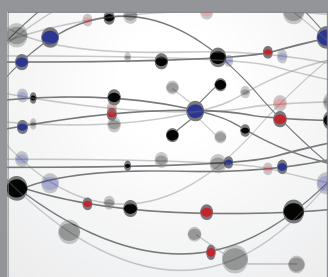

The Scientific World Journal

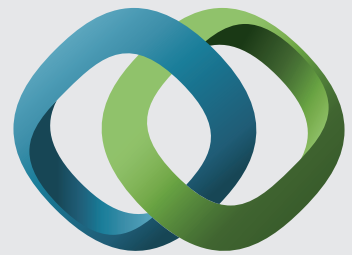

\section{Hindawi}

Submit your manuscripts at

http://www.hindawi.com
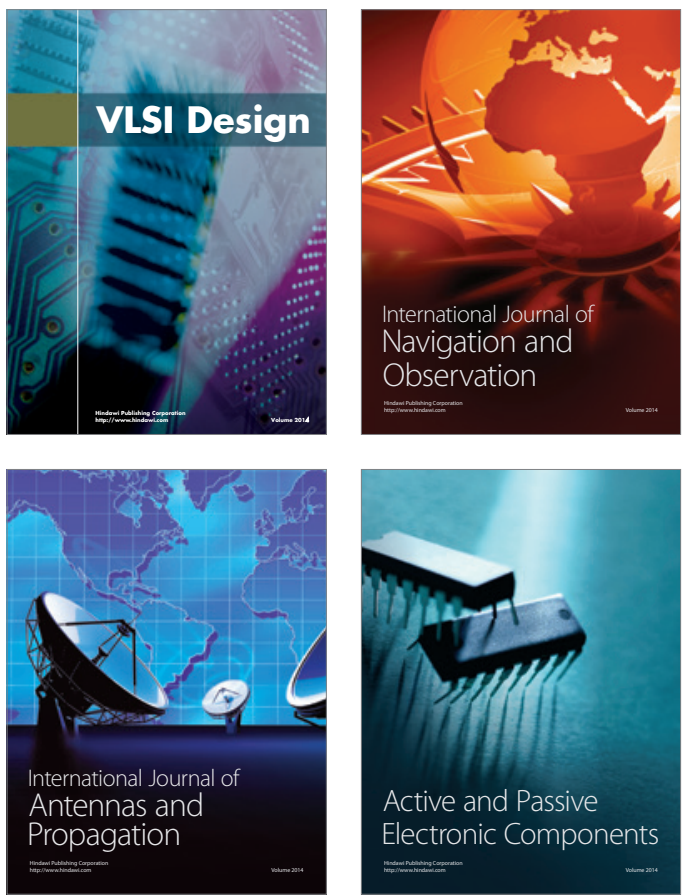
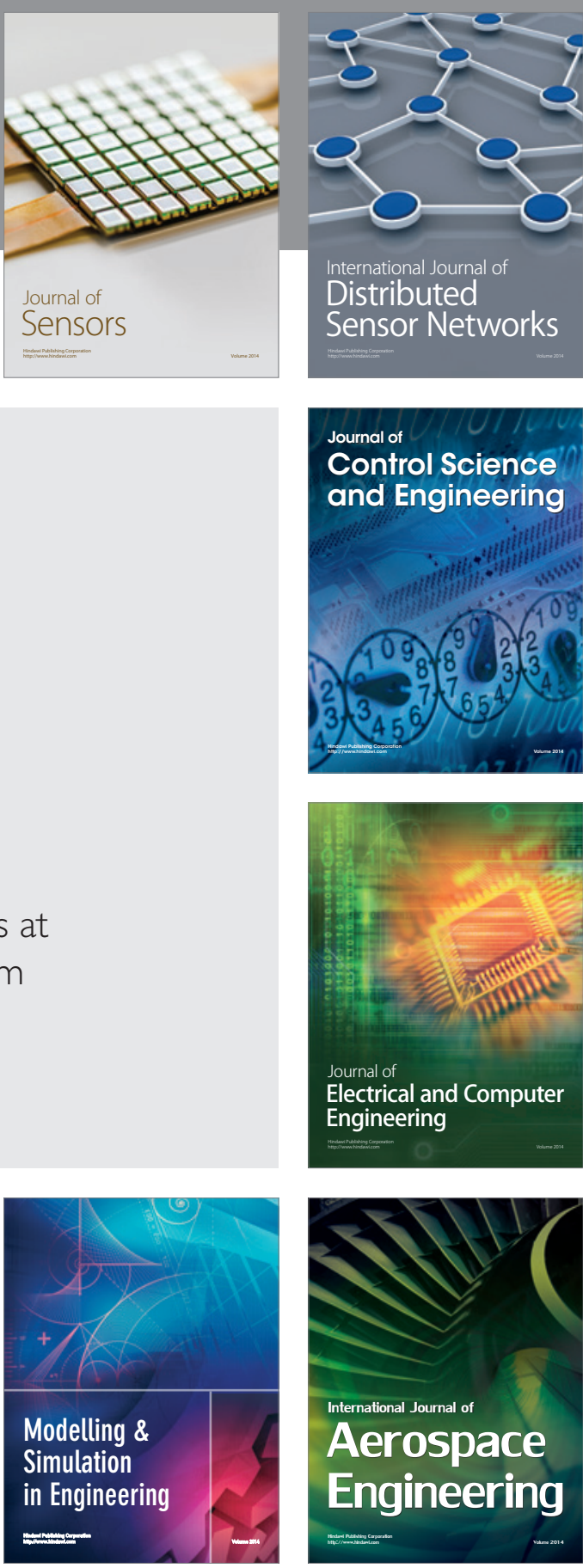

International Journal of

Distributed

Sensor Networks

Journal of

Control Science

and Engineering
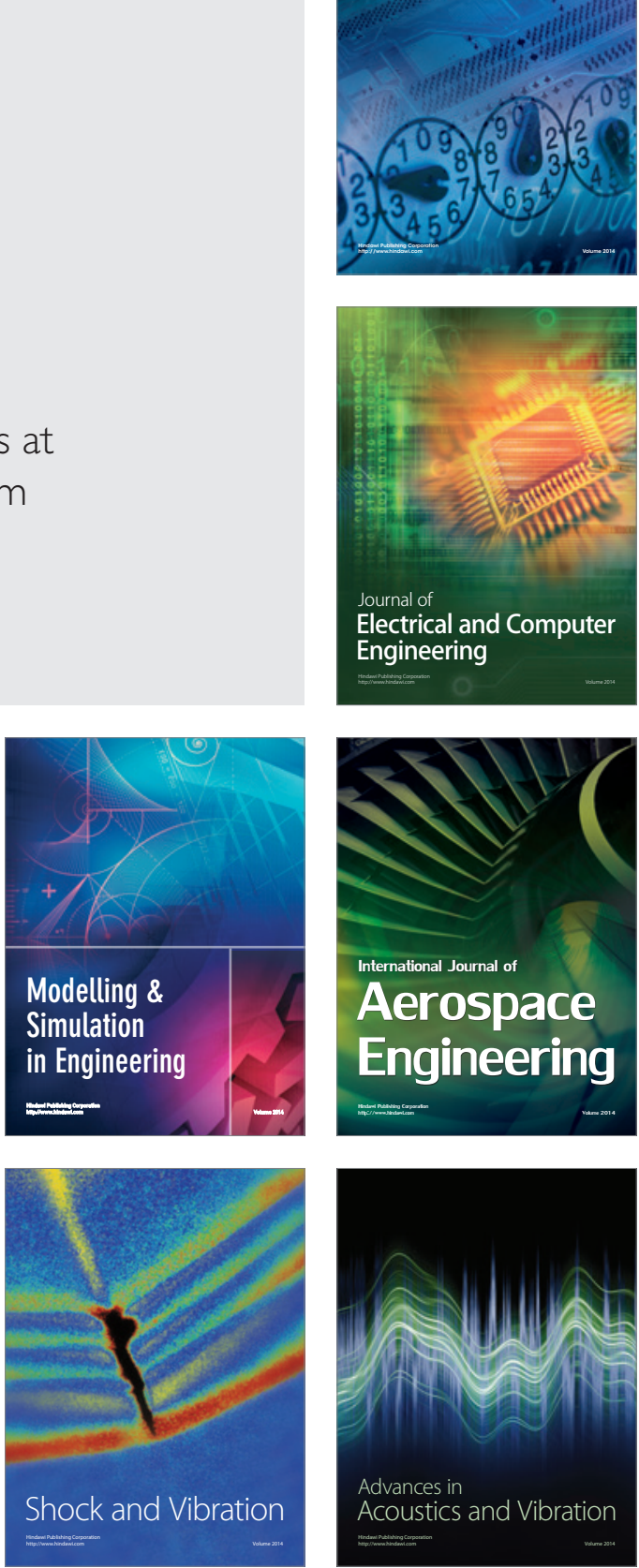